\title{
MELHORAMENTO GENÉTICO DAS PROPRIEDADES DO CARVÃO VEGETAL DE Eucalyptus ${ }^{1}$
}

\author{
Maria Carolina Gaspar Botrel ${ }^{2}$, Paulo Fernando Trugilho ${ }^{3}$, Sebastião Carlos da Silva Rosado ${ }^{3}$ José \\ Reinaldo Moreira da Silva ${ }^{3}$
}

\begin{abstract}
RESUMO - Os objetivos deste trabalho foram estimar os parâmetros fenotípicos, e genotípicos, e ganhos genéticos para as propriedades do carvão vegetal e selecionar clones de Eucalyptus, por meio de características do carvão vegetal, visando à otimização da sua produção e qualidade. Foram utilizados nove clones híbridos de Eucalyptus cultivados no Município de Taiobeiras, Norte de Minas Gerais. O delineamento experimental utilizado foi o inteiramente casualizado, com três repetições. Os parâmetros fenotípicos e genéticos foram estimados a partir dos quadrados médios das características avaliadas no carvão vegetal. Estimou-se o ganho genético de todas as características que apresentaram significância. O efeito de clone foi significativo quanto ao rendimento gravimétrico do carvão, rendimento em carbono fixo e densidade básica relativa aparente do carvão. O ganho genético previsto para o rendimento gravimétrico do carvão foi de $4,51 \%$ na seleção dos clones FGA-30 e FGA-49 e de 4,16\% para o rendimento em carbono fixo na seleção dos clones FGA-30 e I-249. Para densidade básica relativa aparente, o ganho genético foi de $0,03 \%$ na seleção dos clones FGA49 e FGA-34.
\end{abstract}

Palavras-chave: Eucalipto, carvão vegetal e seleção.

\section{GENETIC IMPROVEMENT OF EUcalyptus CHARCOAL PROPERTIES}

\begin{abstract}
The objectives of this work were to estimate the phenotypic and genetic parameters and genetic gains for charcoal properties, as well as to select Eucalyptus clones on the basis of charcoal characteristics. A total of nine Eucalyptus clones, cultivated in the region of Taiobeiras, Northern Minas Gerais, Brazil, were analyzed. Three-meter logs were used for specimen preparation. The experiment was arranged in a complete randomized design, with three repetitions. The phenotypic and genetic parameters were estimated using the expected mean squares. The genetic gain was estimated for the characteristics showing significant differences. The clone effect was significant for charcoal yield, carbon yield and apparent relative density. The obtained genetic gain predicted for charcoal yield was $4.51 \%$ when the clones FGA-30 and FGA-49 were selected, and $4.16 \%$ for carbon yield when the clones FGA-30 e I-249 were selected. The genetic gain was $0.03 \%$ for relative apparent density, for the selection of the clones FGA-49 e FGA-34.
\end{abstract}

Keywords: Eucalyptus, charcoal and selection.

\footnotetext{
${ }^{1}$ Recebido em 09.04.2006 e aceito para publicação em 03.12.2006.

${ }^{2}$ Engenheira Florestal, Mestre. E-mail: <carolinabotrel@yahoo.com.br>.

${ }^{3}$ Departamento de Ciências Florestais da Universidade Federal de Lavras (UFLA). E-mail: <trugilho@ufla.br>; <scrosado@ufla.br>; jreinaldo@ufla.br>.
} 


\section{INTRODUÇÃO}

Dentre as diversas utilizações da madeira, a produção de carvão vegetal sempre ocupou, e ainda ocupa, posição de destaque nas principais empresas de reflorestamento no Brasil. O carvão tem posição de destaque na economia brasileira e, principalmente, na economia do Estado de Minas Gerais, já que seu consumo representa $66,7 \%$ do total demandado no restante do país. A maior demanda por esse insumo é no setor siderúrgico, o qual é responsável por uma área reflorestada com eucalipto de aproximadamente 1,2 milhão de hectares que produzem, juntamente com a floresta nativa, em torno de 26,2 milhões de metros cúbicos de carvão, contribuindo para a produção de cerca de 9,5 milhões de toneladas de ferro-gusa (SBS, 2005).

Os plantios das empresas siderúrgicas não são capazes de, em regime sustentável, atender às suas demandas por carvão vegetal oriundo de florestas plantadas. Somente no Estado de Minas Gerais, a área anual de plantio atinge $30 \mathrm{mil}$ hectares, quando deveriam ser plantados 150 mil hectares. (IMPORTÂNCIA..., 2001).

Os progressos alcançados, principalmente em termos de produtividade da madeira, têm sido expressivos. Nota-se que existe grande interesse em realizar trabalhos conjuntos entre os setores florestal e industrial na busca de matéria-prima que apresente elevada produtividade e qualidade. De modo geral, podem-se melhorar, modificar, controlar ou minimizar os fatores que afetam a qualidade da madeira, por meio de tratos silviculturais e de seleção e melhoramento genético (XAVIER et al., 1997).

A bibliografia que trata da seleção de material superior para fins de carvão vegetal ainda é insuficiente. Também, são necessários os estudos que envolvem a qualidade da madeira, a partir de características físicas, químicas e anatômicas, desejáveis à conversão energética. Seus objetivos são otimizar a produção e aliviar a pressão das florestas nativas. Estudos que consideram o desempenho do produto também são muito importantes, já que o aprimoramento da matéria-prima, em conjunto com o produto obtido, proporciona respostas mais efetivas.

Assim, o objetivo do presente trabalho foi estimar os parâmetros fenotípicos e genotípicos e os ganhos genéticos das propriedades do carvão vegetal produzido, bem como selecionar clones de Eucalyptus por meio de características do carvão vegetal, visando à otimização da produção e da qualidade.

\section{MATERIAL E MÉTODOS}

Neste estudo foram utilizados nove clones híbridos de Eucalyptus spp. com 78 meses, plantados no espaçamento 2 x $3 \mathrm{~m}$. Os clones foram provenientes da Empresa Carvovale, localizados no Município de Taiobeiras, região Norte de Minas Gerais (latitude de 1548'S, longitude de $42^{\circ} 13^{\prime} \mathrm{W}$ e altitude média de $1.090 \mathrm{~m}$ ), com temperatura média anual de $24^{\circ} \mathrm{C}$ e precipitação média anual de $827 \mathrm{~mm}$.

Foram coletadas três árvores por clone, cujas médias dos diâmetros a 1,30 m, das alturas totais e dos volumes são apresentadas no Quadro 1 .

Quadro 1 - Valores médios do diâmetro a 1,30 m (DAP), altura total (HT) e volume de clones de Eucalyptus spp. avaliados aos 78 meses de idade

Table 1 - Mean values of diameter at breast height $(1.30 \mathrm{~m})$, total height and volume for 78-month-old Eucalyptus spp. clones

\begin{tabular}{|c|c|c|c|c|}
\hline Clone & Cruzamentos & $\mathrm{DAP}(\mathrm{cm})$ & $\mathrm{HT}(\mathrm{m})$ & Volume $\left(\mathrm{m}^{3}\right)$ \\
\hline FGA-49 & E. tereticornis $\mathrm{x}$ E. pellita & 16,9 & 23,1 & 0,2500 \\
\hline FGA-35 & E. tereticornis $\mathrm{x}$ E. pellita & 14,9 & 23,7 & 0,2018 \\
\hline FGA-34 & E. tereticornis $\mathrm{x}$ E. pellita & 16,9 & 25,1 & 0,2865 \\
\hline FGA-30 & E. tereticornis $\mathrm{x}$ E. pellita & 20,4 & 24,2 & 0,3527 \\
\hline FGA-50 & E. tereticornis $\mathrm{x}$ E. pellita & 17,6 & 25,0 & 0,2781 \\
\hline $\mathrm{I}-953$ & E. camaldulensis $\mathrm{x}$ E. grandis & 14,7 & 22,9 & 0,1891 \\
\hline I-601 & E. camaldulensis $\mathrm{x}$ E. grandis & 16,5 & 26,9 & 0,2336 \\
\hline $\mathrm{I}-380$ & E. camaldulensis $\mathrm{x}$ E. grandis & 15,7 & 25,7 & 0,2224 \\
\hline $\mathrm{I}-249$ & E. camaldulensis $\mathrm{x}$ E. grandis & 15,9 & 22,6 & 0,1891 \\
\hline
\end{tabular}

R. Árvore, Viçosa-MG, v.31, n.3, p.391-398, 2007 
Após o abate das árvores, retirou-se a primeira tora de $3 \mathrm{~m}$ de comprimento. As toras foram transportadas para a Unidade Experimental de Desdobro e Secagem da Madeira, do Departamento de Ciências Florestais da Universidade Federal de Lavras (DCF-UFLA), onde se retirou um pranchão central de $8 \mathrm{~cm}$ de espessura. Esse pranchão foi levado ao Laboratório de Usinagem da Madeira (DCF-UFLA) para a retirada dos corposde-prova necessários à carbonização da madeira.

As carbonizações foram realizadas em uma mufla elétrica adaptada, conforme mostrado na Figura 1. O controle da taxa de aquecimento foi manual, com incrementos de $50^{\circ} \mathrm{C}$ a cada $30 \mathrm{~min}$, correspondendo a uma média de $1,67^{\circ} \mathrm{C}$ por minuto. A temperatura inicial foi de $100{ }^{\circ} \mathrm{C}$ e a temperatura máxima, $450{ }^{\circ} \mathrm{C}$, permanecendo estabilizada por um período de $30 \mathrm{~min}$. O tempo total de carbonização foi de $4 \mathrm{~h}$. Foram usados em cada ensaio aproximadamente $300 \mathrm{~g}$ de madeira. Esse material foi previamente secado em estufa, à temperatura de $(105 \pm 2)^{\circ} \mathrm{C}(\mathrm{ABNT}, 2003)$.

Após cada carbonização, foram determinados o rendimento gravimétrico em carvão, em líquido pirolenhoso, e o rendimento em gases não-condensáveis pela diferença, todos em relação à massa seca da madeira. Foi determinado também o rendimento em carbono fixo.

A

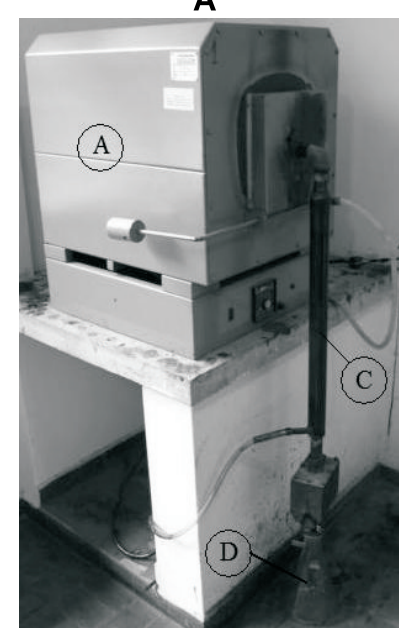

Figura 1 - Esquema do aparato utilizado nas carbonizações. Em que $\mathrm{A}=$ forno elétrico, $\mathrm{B}=$ Cápsula de carbonização, $\mathrm{C}=$ condensador metálico com resfriamento a água e $\mathrm{D}=$ frasco coletor (kitasato).

Figure 1 - Equipment used for wood carbonization. Where, A- electric kiln, $B$-carbonization capsule, $C$-water cooled metal condenser and $D$-collecting bottle.
Foi realizada a análise química imediata do carvão para determinar os teores de material volátil, de cinzas e de carbono fixo, por meio da Norma NBR 8112 (ABNT, 1983).

A densidade básica relativa aparente do carvão vegetal foi determinada pelo método hidrostático, por meio da imersão em água, conforme descrito por Vital (1984).

A análise estatística das características do carvão vegetal foi realizada ao empregar o "Software Genes", versão 2.1, 2004. Para as análises de variância dessas características, seguiu-se o delineamento inteiramente casualizado, conforme o modelo estatístico apresentado na equação 1 .

$$
\mathrm{Y}_{\mathrm{ij}}=\mu+\mathrm{C}_{\mathrm{i}}+\mathrm{e}_{\mathrm{ij}}
$$

em que:

$\mathrm{Y}_{\mathrm{ij}}=$ observaçãodoi-ésimoclonenaj-ésima repetição(ramete); $\mu=$ média geral;

$\mathrm{C}_{\mathrm{i}}=$ efeito do i-ésimo clone $(\mathrm{i}=1,2, \ldots, 9)$, efeito fixo; $\mathrm{e}$ $\mathrm{e}_{\mathrm{ij}}=$ erro experimental associado à observação $\mathrm{Y}_{\mathrm{ij}}$.

A estrutura de análise de variância, seguindo o modelo estatístico da equação 1, é apresentada no Quadro 2.

Pela esperança dos quadrados médios da análise de variância, foram estimados os seguintes parâmetros genéticos, fenotípicos e ambientais, conforme Cruz (1997):

a) Variância fenotípica $\hat{\sigma}_{\mathrm{f}}^{2}=\frac{Q_{1}}{r}$

b) Variância ambiental $\hat{\sigma}_{e}^{2}=Q_{2}$

c) Componente quadrático genotípico $\hat{\phi}_{c}^{2}=\frac{Q_{1}-Q_{2}}{r}$

d)Coeficiente de determinação genotípica $h_{c}^{2}(\%)=\frac{\hat{\phi}_{c}^{2}}{\hat{\sigma}_{F}^{2}} \times 100$

e) Coeficiente de variação genética $C V c \%=\frac{\sqrt{\hat{\phi}_{c}^{2}}}{\text { média }} \times 100$ f) Coeficiente de variação experimental $C V e \%=\frac{\sqrt{\hat{\sigma}_{e}^{2}}}{\text { média }} \times 100$

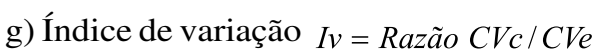

h) Ganho esperado com a seleçãoGanho $=i \mathrm{x} \sqrt{\hat{\phi}_{c}^{2}} \mathrm{x}$ $\sqrt{h_{c}^{2}} \times 100$

em que: $i=$ índice de seleção

R. Árvore, Viçosa-MG, v.31, n.3, p.391-398, 2007 
Quadro 2 - Estrutura da análise de variância das características do carvão vegetal

Table 2 - Variance analysis for charcoal characteristics

\begin{tabular}{lcccc}
\hline $\begin{array}{l}\text { Fonte de } \\
\text { variação }\end{array}$ & $\begin{array}{c}\text { Grau de } \\
\text { liberdade }\end{array}$ & E(Q.M.) & Q.M. & $\mathrm{F}$ \\
\hline Clone & $(\mathrm{C}-1)$ & $\hat{\sigma}_{e}^{2}+r \phi_{c}^{2}$ & $\mathrm{Q}_{1}$ & $\mathrm{Q}_{1} \div \mathrm{Q}_{2}$ \\
Erro & $(\mathrm{C}-1) \mathrm{r}$ & $\hat{\sigma}_{e}^{2}$ & $\mathrm{Q}_{2}$ & \\
\hline
\end{tabular}

Os ganhos foram previstos pela seleção de dois clones em nove, seleção de $22,22 \%$, que padroniza um índice de 1,3426, conforme o que apresentaram Cotteril e Dean (1990); para a comparação múltipla das médias, utilizou-se o teste de Scott-Knott a 5\% de significância.

\section{RESULTADOS E DISCUSSÃO}

A avaliação dos rendimentos obtidos no processo de carbonização é de extrema importância quando o material estudado se destina à produção de carvão vegetal. No Quadro 3 é apresentado o resumo da análise de variância das características de rendimento do processo de carbonização. Pode-se observar que somente as características rendimento gravimétrico em carvão (RGC) e rendimento em carbono fixo (RCF) apresentaram efeito de clone significativo $(\mathrm{p} \leq 0,01)$. Para o rendimento em líquido pirolenhoso (RLP) e gases não-condensáveis (RGNC), o efeito de clone foi não-significativo. Nunes e Andrade (2000), ao compararem o rendimento em gases condensáveis e o rendimento em gases não-condensáveis de Euterpe edulis e Eucalyptus urophylla, concluíram que a uma temperatura final de $300^{\circ} \mathrm{C}$ essas espécies apresentavam comportamentos diferenciados com relação a essas características. Porém, em temperatura final de $500^{\circ} \mathrm{C}$ não houve diferenças significativas em RLP e RGNC, demostrando que essas variáveis são extremamente influenciadas por temperaturas finais mais elevadas. Assim como ocorreu neste trabalho, Trugilho et al. (2005), estudando clones de Eucalyptus spp, encontraram diferenças significativas em RGC e RCF e não-significativas em RLP e RGNC.

O rendimento em carbono fixo médio foi de $25,97 \%$. Esse valor foi semelhante aos $27,20 \%$, encontrados por Vale et al. (1996) ao trabalharem com Eucalyptus grandis a uma temperatura máxima de carbonização de $463^{\circ} \mathrm{C}$. Andrade et al. (2004) relataram que o rendimento em carbono fixo é o principal parâmetro a ser considerado na análise termogravimétrica de material lignocelulósico. O rendimento em carbono fixo envolve, simultaneamente, características de produtividade e de qualidade relacionadas ao carvão vegetal (ANDRADE, 1989).

Quadro 3 - Resumo da análise de variância e estimativa dos parâmetros genéticos do rendimento gravimétrico em carvão (RGC), rendimento em carbono fixo (RCF), rendimento em líquido pirolenhoso (RLP) e rendimento em gases não-condensáveis (RGCN), em clones de Eucalyptus spp., aos 78 meses de idade

Table 3 - Summary of variance analysis and estimated genetic parameters for charcoal yield, carbon yield and non-condensed gases yield, for 78-month-old Eucalyptus spp. clones

\begin{tabular}{|c|c|c|c|c|c|}
\hline \multirow[t]{2}{*}{ Fontes de Variação } & \multirow[t]{2}{*}{ Graus de Liberdade } & \multicolumn{4}{|c|}{ Quadrados Médios } \\
\hline & & $\operatorname{RGC}(\%)$ & $\mathrm{RCF}(\%)$ & RLP (\%) & RGNC (\%) \\
\hline Clones & 8 & $6,92 * *$ & $2,55 * *$ & $14,61^{\mathrm{ns}}$ & $7,78^{\mathrm{ns}}$ \\
\hline$\underline{\text { Resíduo }}$ & 18 & 1,55 & 0,32 & 8,53 & 5,23 \\
\hline Média & & 35,03 & 25,97 & 45,44 & 19,53 \\
\hline $\mathrm{CV}_{\mathrm{e}}(\%)$ & & 3,56 & 2,20 & 6,43 & 11,71 \\
\hline $\mathrm{CV}_{\mathrm{c}}(\%)$ & & 3,82 & 3,32 & - & - \\
\hline$\sigma_{f}^{2}$ & & 2,31 & 0,85 & - & - \\
\hline$\sigma_{e}^{2}$ & & 0,52 & 0,11 & - & - \\
\hline$\phi_{\mathrm{c}}^{2}$ & & 1,79 & 0,74 & - & 一 \\
\hline$\overline{\mathrm{h}_{\mathrm{c}}^{2}}$ & & 77,53 & 87,27 & - & - \\
\hline $\mathrm{CV}_{\mathrm{c}} / \mathrm{CV}_{\mathrm{e}}$ & & 1,07 & 1,51 & - & - \\
\hline Ganho & & 1,58 & 1,08 & - & - \\
\hline Ganho (\%) & & 4,51 & 4,16 & - & - \\
\hline
\end{tabular}

${ }^{\mathrm{ns}}$ não-significativo; **significativo, pelo teste de $\mathrm{F}(\mathrm{p} \leq 0,01) ; \mathrm{CV}_{\mathrm{e}}$ : coeficiente de variação experimental; $\mathrm{CV}_{\mathrm{c}}$ : coeficiente de variação genética; $\sigma_{\mathrm{f}}^{2}$ : variação fenotípica; $\sigma_{\mathrm{e}}^{2}$ : variação ambiental; $\phi_{\mathrm{c}}^{2}:$ componente quadrático genotípico; $\mathrm{h}_{\mathrm{c}}{ }_{\mathrm{c}}$ : coeficiente de determinação genotípica; $\mathrm{CV}_{c} / \mathrm{CV}_{e}$ : índice de variação; e Ganho (\%): ganho genético esperado com a seleção de dois clones.

R. Árvore, Viçosa-MG, v.31, n.3, p.391-398, 2007 
O valor médio do rendimento gravimétrico em carvão foi de $35,03 \%$, sendo essa média superior às relatadas por Nunes e Andrade (2000) e Santiago e Andrade (2005), que encontraram rendimentos gravimétricos em carvão de $26,91 \%$ e $25,20 \%$ em Eucalyptus urophylla, respectivamente. Os valores deste trabalho evidenciam o potencial destaa população de clones para participar de programas de seleção e hibridação que visam à produção de energia a partir da biomassa.

A relação $\mathrm{CV}_{\mathrm{c}} / \mathrm{CV}_{\mathrm{e}}$ para RGC e RCF foi de $1,07 \mathrm{e}$ 1,51 , respectivamente. Essa condição é desejável no processo de seleção, já que, dessa forma, a variação genética supera a variação ambiental. Ainda no Quadro 3 , os valores obtidos da herdabilidade para a característica rendimento gravimétrico em carvão foram de 77,53\% e $87,27 \%$, em rendimento em carbono fixo. A herdabilidade, por expressar a proporção da variação que é atribuída a diferenças genéticas entre os indivíduos, é um parâmetro de grande importância. Dessa forma, pode-se inferir que essas características se encontram sob um forte controle genético, respondendo positivamente à seleção.

Os baixos coeficientes de variação ambiental encontrados dessas características, de 3,56\% para RGC e 2,20\% para RCF, constatam a acuracidade dos dados e, com isso, a confiança nos parâmetros genéticos estimados.

Os valores dos coeficientes de herdabilidade e das variâncias genéticas permitem obter ganhos de $4,51 \%$ para rendimento gravimétrico em carvão e $4,16 \%$ para rendimento em carbono fixo. Esses ganhos foram obtidos pela seleção dos dois clones superiores, dentre os nove estudados, resultado de uma seleção de $22,22 \%$, padronizando um índice de seleção de 1,3426, conforme os dados relatados por Cotteril e Dean (1990).

Os valores médios e o teste de comparação múltipla das características de rendimento do processo de carbonização são apresentados no Quadro 4. Os clones com os maiores rendimentos gravimétricos do carvão foram FGA-30 e FGA-49, com os respectivos valores médios de $36,99 \%$ e $36,97 \%$. Para RCF, os clones selecionados foram os FGA-30, com 27,36\%, e I-249, $\operatorname{com} 26,83 \%$.

No Quadro 5, observa-se que, dentre as análises realizadas no carvão vegetal, somente a densidade básica relativa aparente (DRA) apresentou efeito significativo de clone ( $\mathrm{p} \leq 0,05)$. Com relação aos teores de material volátil, de cinzas e de carbono fixo, o efeito de clone foi não significativo. Esse resultado já era esperado, visto que tais características são muito influenciadas pela temperatura final de carbonização. O valor médio de $0,336 \mathrm{~g} / \mathrm{cm}^{3}$ da DRA está de acordo com o encontrado em outros trabalhos (BRITO et al., 1987; TRUGILHO, 1995; ANDRADE e MACHADO, 2004). Em relação à qualidade de carvões para fins siderúrgicos, evidenciase a importância da sua densidade, pois essa característica está diretamente relacionada à resistência mecânica do carvão. ADRA relaciona-se com importantes aspectos operacionais e produtivos das usinas siderúrgicas. Além disso, quanto maior a referida densidade, menores os custos de transporte e de armazenamento do carvão e, simultaneamente, melhor o aproveitamento do volume útil do alto-forno siderúrgico, e isso permite o aumento da sua produtividade em determinado espaço de tempo.

Quadro 4 - Valores médios de rendimento gravimétrico do carvão (RGC), rendimento em carbono fixo (RCF), rendimento em líquido pirolenhoso (RLP) e rendimento em gases não-condensáveis (RGNC) de clones de Eucalyptus spp., aos 78 meses de idade

Table 4-Mean values of charcoal yield, carbon yield, pyroligeneous liquid yield and non-condensed gases yield for 78month-old Eucalyptus spp. clones

\begin{tabular}{|c|c|c|c|c|}
\hline Clone & RGC (\%) & $\mathrm{RCF}(\%)$ & RLP $(\%)$ & RGNC (\%) \\
\hline FGA-49 & $36,97 \mathrm{a}$ & 26,75 a & $43,40 \mathrm{a}$ & $19,63 \mathrm{a}$ \\
\hline FGA-35 & $35,23 \mathrm{a}$ & $24,97 \quad b$ & $46,64 \mathrm{a}$ & $18,13 \mathrm{a}$ \\
\hline FGA-34 & $33,37 \quad b$ & $24,90 \quad b$ & $46,61 \mathrm{a}$ & $20,02 \mathrm{a}$ \\
\hline FGA-30 & 36,99 a & 27,36 a & $44,64 \mathrm{a}$ & 18,36 a \\
\hline I-953 & $36,14 \mathrm{a}$ & $26,65 \mathrm{a}$ & $43,00 \mathrm{a}$ & 20,86 a \\
\hline I- 601 & $33,47 \quad b$ & $25,43 \quad b$ & $44,26 \mathrm{a}$ & 22,27 a \\
\hline I-380 & $33,18 \quad b$ & $25,42 \quad b$ & 49,69 a & 17,13 a \\
\hline I-249 & $35,65 \mathrm{a}$ & 26,83 a & $43,65 \mathrm{a}$ & 20,69 a \\
\hline FGA-50 & $34,27 \quad b$ & $25,40 \quad b$ & $47,08 \mathrm{a}$ & 18,65 \\
\hline
\end{tabular}

*Médias seguidas de mesma letra na coluna não diferem entre si, pelo teste de agrupamento de Scott-Knott $(\mathrm{p} \leq 0,05)$. 
Quadro 5 - Resumo da análise de variância e estimativa dos parâmetros genéticos de teor de materiais voláteis (TMV), teor de cinzas no carvão (TCz), teor de carbono fixo (TCF) e densidade relativa aparente (DRA), em clones de Eucalyptus spp., aos 78 meses de idade

Table 5 -Summary of variance analysis and estimated genetic parameters for volatile materials, ash content, fixed carbon content and apparent relative density, for 78-month-old Eucalyptus spp. clones

\begin{tabular}{|c|c|c|c|c|c|}
\hline \multirow[t]{2}{*}{ Fontes de variação } & \multirow[t]{2}{*}{ Graus de Liberdade } & \multicolumn{4}{|c|}{ Quadrados Médios } \\
\hline & & $\operatorname{TMV}(\%)$ & $\mathrm{TCz}(\%)$ & $\mathrm{TCF}(\%)$ & DRA $\left(\mathrm{g} / \mathrm{cm}^{3}\right)$ \\
\hline Clones & 8 & 7,54 ns & $0,01^{\mathrm{ns}}$ & $7,56 \mathrm{~ns}$ & $0,004 *$ \\
\hline Resíduo & 18 & 7,24 & 0,0098 & 7,23 & 0,002 \\
\hline Média & & 25,50 & 0,25 & 74,25 & 0,336 \\
\hline$\overline{\mathrm{CV}}(\%)$ & & 10,55 & 40,29 & 3,62 & 11,174 \\
\hline $\mathrm{CV}_{\mathrm{c}}^{\mathrm{e}}(\%)$ & & - & - & - & 8,391 \\
\hline$\sigma_{f}^{2}$ & & - & - & - & 0,0013 \\
\hline$\sigma_{e}^{2}$ & & - & - & - & 0,0005 \\
\hline$\phi_{c}^{{ }^{e}}$ & & - & - & - & 0,0008 \\
\hline$\overline{\mathrm{h}^{2}{ }_{\mathrm{c}}}$ & & - & - & - & 62,853 \\
\hline $\mathrm{CV}_{\mathrm{c}} / \mathrm{CV}_{\mathrm{e}}$ & & - & - & - & 0,75 \\
\hline Ganho & & - & - & - & 0,030 \\
\hline Ganho(\%) & & - & - & - & 8,93 \\
\hline
\end{tabular}

${ }^{n s}$ não-significativo; *significativo, pelo teste de $\mathrm{F}(\mathrm{p} \leq 0,05) ; \mathrm{CV}_{\mathrm{e}}$ : coeficiente de variação experimental; $\mathrm{CV}$ : coeficiente de variação genética; $\sigma_{\mathrm{f}}^{2}$ : variação fenotípica; $\sigma_{\mathrm{e}}^{2}:$ variação ambiental; $\phi_{\mathrm{c}}^{2}:$ componente quadrático genotípico; $\mathrm{h}_{\mathrm{c}}^{2}$ : coeficiente de determinação genotípica; CV ${ }_{\mathrm{c}}$ $\mathrm{CV}_{\mathrm{e}}$ : índice de variação; e Ganho $(\%)$ : ganho genético esperado com a seleção de dois clones.

O coeficiente de variação experimental da DRA foi de $11,17 \%$, valor esse semelhante ao encontrado por Trugilho et al. (2001), que trabalharam com clones de E. saligna.

Mesmo que o teste de Scott-Knott (Quadro 6) não tenha sido capaz de detectar diferenças significativas, a 5\% de significância, entre os clones na densidade básica relativa aparente, a análise de variância indicou efeito de clone significativo quanto a essa característica e permitiu estimar os parâmetros genéticos que auxiliassem o processo de obtenção de ganhos genéticos.

Ao selecionar os dois clones de maior valor para essa característica, obteve-se um aumento, em relação à média, de $0,030 \mathrm{~g} / \mathrm{cm}^{3}$, o que corresponde a um acréscimo de 8,93\% na densidade básica relativa aparente.

No Quadro 6 são apresentados os valores médios e o teste de comparação múltipla d as análises químicas imediatas do carvão vegetal e da densidade relativa aparente.

Madeiras de densidades mais elevadas produzem carvão com maior densidade aparente. Isto se verifica pelos clones selecionados, FGA-49 e FGA-34, que apresentaram carvões com densidade básica relativa aparente de 0,402 e $0,365 \mathrm{~g} / \mathrm{cm}^{3}$, respectivamente.

Quadro 6 - Valores médios do teor de materiais voláteis (TMV), teor de cinzas no carvão, teor de carbono fixo (TCF) e densidade relativa aparente (DRA) de clones de Eucalyptus spp., aos 78 meses de idade

Table 6-Mean values of volatile materials, ash content, fixed carbon content and apparent relative density for 78-monthold Eucalyptus spp. clones

\begin{tabular}{ccccc}
\hline Clones & TMV $(\%)$ & TCz $(\%)$ & TCF $(\%)$ & DRA $\left(\mathrm{g} / \mathrm{cm}^{3}\right)$ \\
\hline FGA-49 & $27,98 \mathrm{a}$ & $0,27 \mathrm{a}$ & $71,74 \mathrm{a}$ & $0,402 \mathrm{a}$ \\
FGA-35 & $26,22 \mathrm{a}$ & $0,16 \mathrm{a}$ & $73,62 \mathrm{a}$ & $0,318 \mathrm{a}$ \\
FGA-34 & $25,57 \mathrm{a}$ & $0,34 \mathrm{a}$ & $74,09 \mathrm{a}$ & $0,365 \mathrm{a}$ \\
FGA-30 & $25,76 \mathrm{a}$ & $0,16 \mathrm{a}$ & $74,07 \mathrm{a}$ & $0,324 \mathrm{a}$ \\
I-953 & $26,34 \mathrm{a}$ & $0,23 \mathrm{a}$ & $73,42 \mathrm{a}$ & $0,280 \mathrm{a}$ \\
I-601 & $23,43 \mathrm{a}$ & $0,31 \mathrm{a}$ & $76,26 \mathrm{a}$ & $0,313 \mathrm{a}$ \\
I-380 & $22,86 \mathrm{a}$ & $0,22 \mathrm{a}$ & $76,93 \mathrm{a}$ & $0,359 \mathrm{a}$ \\
I-249 & $24,87 \mathrm{a}$ & $0,20 \mathrm{a}$ & $74,93 \mathrm{a}$ & $0,333 \mathrm{a}$ \\
FGA-50 & $26,48 \mathrm{a}$ & $0,32 \mathrm{a}$ & $73,20 \mathrm{a}$ & $0,325 \mathrm{a}$ \\
\hline
\end{tabular}

*médias seguidas de mesma letra na coluna não diferem entre si, pelo teste de agrupamento de Scott-Knott $(\mathrm{p} \leq 0,05)$.

R. Árvore, Viçosa-MG, v.31, n.3, p.391-398, 2007 


\section{CONCLUSÕES}

O rendimento gravimétrico de carvão, o rendimento de carbono fixo e a densidade básica relativa aparente apresentaram efeito significativo de clones.

Os valores de herdabilidades foram considerados altos para rendimento gravimétrico de carvão, rendimento de carbono fixo e densidade básica relativa aparente, indicando forte controle genético na expressão de tais características.

O ganho genético calculado do rendimento gravimétrico de carvão foi de $4,51 \%$, na seleção dos clones FGA-30 e FGA-49. No rendimento de carbono fixo, o ganho calculado foi de $4,16 \%$, na seleção dos clones FGA-30 e I-249. Quanto à densidade básica relativa aparente, o ganho genético foi de $0,03 \%$, na seleção dos clones FGA-49 e FGA-34.

Visando à produção de carvão vegetal os clones FGA-30 e FGA-49 foram os de maior destaque, os quais devem ser indicados para multiplicação vegetativa e plantio comercial.

\section{REFERÊNCIAS}

ANDRADE, A. M. Influência da casca de Eucalyptus grandis W. Hill ex Maiden no rendimento e qualidade de carvão vegetal. 1989. 86f. Dissertação (Mestrado em Ciência Florestal) - Universidade Federal de Viçosa, Viçosa, MG, 1989.

ANDRADE, A. M. et al. Pirólise de resíduos do coco-da-baía (Cocos nucifera Limn) e análise do carvão vegetal. Revista Árvore, v.28, n.5, p.707-714, 2004.

ANDRADE, A. M.; MACHADO, F. S.

Comparação entre as propriedades físicas e mecânicas dos finos de carvão vegetal e de carvão mineral, para injeção nas ventaneiras de alto-fornos siderúrgicos. Biomassa e Energia, v.1, n.3, p.273-279, 2004.

ASSOCIAÇÃO BRASILEIRA DE NORMAS TÉCNICAS - ABNT. Normas técnicas NBR 11941 - Madeira - Determinação da densidade básica. Rio de Janeiro: 2003. 6p.

ASSOCIAÇÃO BRASILEIRA DE NORMAS TÉCNICAS - ABNT. Normas técnicas NBR 8633. Brasília: 1983. Não Paginado.
BRITO, J. O.; TOMAZELLO FILHO, M.; SALGADO, A. L. Produção e caracterização do carvão vegetal de espécies e variedades de bambu. IPEF, n.36, p.13-17, 1987.

COTTERIL, P. P.; DEAN, C. A. Successful tree breeding with index selection. Melbourne: CSIRO, 1990. 80p.

CRUZ, C. D. Programa genes: aplicativo computacional em genética e estatística. Viçosa, MG: Universidade Federal de Viçosa, 1997. 442p.

NUNES, W. H.; ANDRADE, A. M. Pirólise do estirpe de Euterpe edulis Martius (Palmiteiro). Floresta e Ambiente, v.7, n.1, p.217-226, 2000.

IMPORTÂNCIA do eucalipto para indústria do carvão vegetal. Revista da Madeira, v.11, n.59, 2001. Disponível em <http:// www.remade.com.br>. Acesso em: 13 dez. 2005.

SANTIAGO, A. R.; ANDRADE, A. M.

Carbonização de resíduos do processamento mecânico da madeira de eucalipto. Ciência Florestal, v.15, n.1, p.1-7. 2005.

SOCIEDADE BRASILEIRADE SILVICULTURASBS. Segmento de carvão vegetal.

Disponível em: <http://www.sbs.org.br/ estatisticas.htm>. Acesso em: 13 dez. 2005.

TRUGilho, P. F. Aplicação de algumas técnicas multivariadas na avaliação da qualidade da madeira e do carvão vegetal de Eucalyptus. 1995, 160f. Tese (Doutorado em Ciência Florestal) - Universidade Federal de Viçosa, Viçosa, MG, 1995.

TRUGILHO, P. F. et al. Avaliação de clones de Eucalyptus para a produção de carvão vegetal. CERNE, v.7, n.2, p.104-114, 2001.

TRUGILHO, P. F. et al. Rendimentos e características do carvão vegetal em função da posição radial de amostragem em clones de Eucalyptus. CERNE, v.11, n.2, p.178-186, 2005.

VALE, A. .; NOGUEIRA, M. V. P.; SILVA, M. A. Rendimento da carbonização e qualidade do carvão vegetal de madeiras do cerrado em comparação ao Eucalyptus grandis. Revista Árvore, v.20, n.1, p.93-99, 1996.

R. Árvore, Viçosa-MG, v.31, n.3, p.391-398, 2007 
VITAL, B. R. Métodos de determinação da densidade da madeira. Viçosa, MG:

Sociedade de Investigações Florestais, 1984. 21p. (Boletim Técnico, 1).
XAVIER, A. et al. Parâmetros genéticos de características de qualidade da madeira em Eucalyptus grandis. Revista Árvore, v.21, n.1, p.71-78, 1997. 